\title{
REFINED ASYMPTOTICS FOR THE SUBCRITICAL KELLER-SEGEL SYSTEM AND RELATED FUNCTIONAL INEQUALITIES
}

\author{
VINCENT CALVEZ AND JOSÉ ANTONIO CARRILLO \\ (Communicated by Walter Craig)
}

\begin{abstract}
We analyze the rate of convergence towards self-similarity for the subcritical Keller-Segel system in the radially symmetric two-dimensional case and in the corresponding one-dimensional case for logarithmic interaction. We measure convergence in the Wasserstein distance. The rate of convergence towards self-similarity does not degenerate as we approach the critical case. As a byproduct, we obtain a proof of the Logarithmic Hardy-Littlewood-Sobolev inequality in the one-dimensional and radially symmetric two-dimensional cases based on optimal transport arguments. In addition we prove that the onedimensional equation is a contraction with respect to Fourier distance in the subcritical case.
\end{abstract}

\section{INTRODUCTION}

We will concentrate on seeking decay rates towards equilibria or self-similarity profiles for aggregation equations with linear diffusion in the fair competition regime. These models describe the evolution of a population of individuals which are diffusing by standard Brownian motion and attracting each other by a pairwise symmetric potential $W(x)$. We focus on a logarithmic interaction potential $W(x)=2 \chi \log |x|$, with $\chi>0$. The Fokker-Planck equation governing the evolution of the probability density function $\rho(t, x)$ associated to this particle system reads as

$$
\frac{\partial \rho}{\partial t}=\nabla \cdot\left[\frac{1}{N} \nabla \rho+2 \chi \rho(\nabla \log |x| * \rho)\right], \quad t>0, \quad x \in \mathbb{R}^{N} .
$$

Due to translational invariance and mass conservation, in the rest of this work we restrict ourselves to zero center of mass probability densities,

$$
\rho(t, x) \geq 0, \quad \int_{\mathbb{R}^{N}} \rho(t, x) d x=1, \quad \int_{\mathbb{R}^{N}} x \rho(t, x) d x=0 .
$$

By fair competition, we mean that the dynamics of (1.1) are driven by a simple dichotomy as in the classical Keller-Segel system in two dimensions [23, 29, 19, 12, the modified Keller-Segel system in one dimension [14, 7] or the Keller-Segel model with suitable nonlinear diffusion in larger dimensions [9. In all these examples there is a critical parameter which makes the distinction between global existence of solutions and finite-time blowup. More precisely, we will discuss the modified

Received by the editors July 16, 2010 and, in revised form, April 12, 2011.

2010 Mathematics Subject Classification. Primary 35B40, 92C17.

(C) 2012 American Mathematical Society 
one-dimensional Keller-Segel equation [14, 7]:

$$
\partial_{t} \rho=\partial_{x x}^{2} \rho+2 \chi \partial_{x}\left(\rho \partial_{x}(\log |x| * \rho)\right), \quad t>0, \quad x \in \mathbb{R},
$$

and the radially symmetric two-dimensional classical Keller-Segel equation:

$$
\partial_{t}(r \rho(t, r))=\frac{1}{2} \partial_{r}\left(r \partial_{r} \rho(t, r)\right)+2 \chi \partial_{r}[\rho(t, r) M[\rho(t)](r)], \quad t>0, \quad r \in \mathbb{R}_{+},
$$

where $M[\rho]$ denotes the cumulative mass of $\rho$ inside balls,

$$
M[\rho](r)=2 \pi \int_{0}^{r} \rho(s) s d s .
$$

Both equations (1.3) and (1.4) exhibit a transition depending on the sensitivity coefficient $\chi$ :

- Subcritical Case. For any $0<\chi<1$, solutions exist globally in time and they approach a unique self-similar solution as $t \rightarrow \infty$; see [19, 12, 5, 14.

- Critical Case. For $\chi=1$, solutions exist globally in time. There are infinitely many stationary solutions with infinite second moment. Solutions having finite initial second moment concentrate in infinite time towards the Dirac mass $\delta_{0}$ [10, 5, 22]. Solutions of infinite initial second moment close enough to a stationary solution converge to it as $t \rightarrow \infty$ [ .

- Supercritical Case. For any $\chi>1$, smooth fast-decaying solutions do not exist globally in time [27, 6, 12, 14.

The critical parameter $\chi=1$ can be obtained from two formal computations at this stage. The evolution of the second moment satisfies in both cases the relation:

$$
\frac{1}{2} \frac{d}{d t} \int_{\mathbb{R}}|x|^{2} \rho(t, x) d x=1-\chi .
$$

This implies that for $\chi>1$ solutions will necessarily blow up before the second moment touches zero. On the other hand, the Keller-Segel equation (1.1) is equipped with a free energy (entropy minus potential energy),

$$
\mathcal{F}[\rho]=\frac{1}{N} \int_{\mathbb{R}^{N}} \rho(x) \log \rho(x) d x+\chi \iint_{\mathbb{R}^{N} \times \mathbb{R}^{N}} \rho(x) \log (|x-y|) \rho(y) d x d y .
$$

It is formally decreasing along the trajectories

$$
\frac{d}{d t} \mathcal{F}[\rho(t)]=-\int_{\mathbb{R}^{N}} \rho(t, x)\left|\nabla\left(\frac{1}{N} \log \rho(t, x)+2 \chi \log |x| * \rho(t, x)\right)\right|^{2} d x .
$$

Moreover, it was shown in [19, 12] that for $\chi<1$ the free energy estimate from above implies an a priori bound in the entropy part of the functional which is at the basis of the construction of global-in-time solutions. This was achieved by using the Logarithmic HLS (Hardy-Littlewood-Sobolev) inequality [4, 16] which relates the entropy and the interaction part of the functional.

Nontrivial equilibrium profiles or critical profiles only exist for the critical parameter $\chi=1$. They are solutions to the following Euler-Lagrange equations:

$$
\begin{aligned}
\rho^{\prime}(x)+2 \rho(x) \partial_{x}(\log |x| * \rho(x)) & =0, \\
\frac{1}{2} r \rho^{\prime}(r)+2 \rho(r) M[\rho](r) & =0,
\end{aligned}
$$

resp. in dimension $N=1$ and in dimension $N=2$ with radial symmetry. In fact, we have an explicit formulation of the stationary states. Once we have reduced by 
symmetries (1.2) to centered probability densities, they are given by dilations of the profile

$$
\mu(x):=\frac{1}{\pi\left(1+|x|^{2}\right)^{N}}, \quad N=1,2,
$$

i.e., by $\mu_{\lambda}(x)=\lambda^{-N} \mu\left(\lambda^{-1} x\right)$ with $\lambda>0$. The set of equilibria coincides with the equality cases in the Logarithmic HLS inequality: given by the profile $\mu$ modulo dilations and translations once restricted to probability densities.

In the subcritical case $\chi<1$, solutions are known to converge to unique selfsimilar profiles [12. For studying convergence towards self-similarity, it is generally useful to rescale the space and time variables in the subcritical regime $\chi<1$. The Keller-Segel system can be rewritten as

$$
\frac{\partial \rho}{\partial t}=\frac{1}{N} \Delta \rho+2 \chi \nabla \cdot[\rho(\nabla \log |x| * \rho)]+\nabla \cdot[x \rho], \quad t>0, \quad x \in \mathbb{R}^{N},
$$

and the free energy is complemented with a quadratic confinement potential:

$$
\mathcal{F}_{\text {resc }}[\rho]=\mathcal{F}[\rho]+\frac{1}{2} \int_{\mathbb{R}^{N}}|x|^{2} \rho(x) d x .
$$

Due to the change of variables, self-similar solutions correspond to equilibrium solutions of (1.10). In fact, it is shown in 12 for $N=2$ and $\chi<1$ that (1.10) admits a unique radially symmetric stationary state satisfying (1.2) that we will denote by $\nu(x)$. This equilibrium state is characterized as the unique minimizer of the rescaled energy functional (1.11) in the class of densities satisfying (1.2). The same argument applies in the one-dimensional case. The rate of convergence towards the equilibrium $\nu(x)$ in $N=2$ for (1.10) in the subcritical case was recently studied in [11] where the same rate as for the heat equation was obtained for small mass.

Let us finally mention that both (1.1) and (1.10) are gradient flows of the free energy functionals (1.5) and (1.11), respectively, when the space of probability measures is endowed with the Euclidean Wasserstein metric $W_{2}$. We refer to the seminal papers 21, 28 and to 1 for a general theory. For instance, we can write (1.1) in short as

$$
\dot{\rho}(t)=-\nabla_{W_{2}} \mathcal{F}[\rho(t)] .
$$

This assertion was made rigorous in [7, where the variational minimizing movement scheme [1] was shown to converge for (1.1). This fact allows us to consider a way of measuring the distance towards equilibrium or self-similarity intimately related to the evolution due to (1.12). In fact, we will show that optimal transport tools are key techniques to describe this behavior at least in the one-dimensional case (1.3) and in the radial case in two dimensions (1.4).

In order to investigate further the bounds of the free energy functional leading to the dichotomy discussed above and the characterization of the critical profiles, the Logarithmic HLS inequality proved in [4, 16] is essential. In Section 2, we show an alternative proof based on optimal transport tools in the one-dimensional case and in the radial case in two dimensions. There is another recent proof of this inequality with sharp constants in the two-dimensional case by fast diffusion flows [15]. The Logarithmic HLS inequality can be restated with our notation as:

Theorem 1.1 (Logarithmic HLS inequality). Assume $N=1,2$. The functional $\mathcal{F}$ defined in (1.5) is bounded from below. The extremal functions are uniquely given 
by the profile $\mu$ in (1.9) up to dilations in the set of probability densities with zero center of mass.

In short, we demonstrate that any critical point of the free energy is in fact a global minimizer. This is a property which holds true for convex functionals, although the functional $\mathcal{F}$ is not displacement convex in the sense of McCann 26].

We stress that the Logarithmic HLS inequality holds true in higher dimensions. However our method of proof fails to work for $N \geq 3$ even in the radially symmetric case. There is no clear obstruction, except for technical reasons.

The ideas behind the proof of the sharp Logarithmic HLS inequality allow us to tackle the rate of convergence in $W_{2}$ by similar methods for the rescaled version (1.10) in one dimension and for radial densities in the two-dimensional case provided $\chi<1$. We prove in Section 3 the following result.

Theorem 1.2 (Long-time asymptotics). Assume that $N=1,2$ and $\chi<1$. In the case $N=2$ assume in addition that the initial data $\rho_{0}$ is radially symmetric. Then, solutions of (1.10) with conserved quantities as in (1.2) converge exponentially fast towards the unique equilibrium configuration $\nu$ in the Euclidean Wasserstein distance. More precisely, the following estimate holds true for solutions of (1.10):

$$
\frac{d}{d t} W_{2}(\rho(t), \nu)^{2} \leq-2 W_{2}(\rho(t), \nu)^{2} .
$$

Surprisingly enough, the rate of convergence that we obtain does not depend on the parameter $\chi$. Our estimate is uniform as long as $\chi$ remains subcritical and is equal to the rate of convergence towards self-similarity for the heat equation. This is due to the fact that the entropy and interaction contributions cancel each other, and only the confinement contribution remains, yielding a uniform estimate. Although convergence is likely to be uniform, notice that the asymptotic profile becomes more and more singular as $\chi \rightarrow 1^{-}$. This is emphasized by the simple second moment identity

$$
\int_{\mathbb{R}^{N}}|x|^{2} \nu(x) d x=1-\chi
$$

Finally, we devote Section 4 to proposing an alternative method of measuring the distance towards self-similarity in the one-dimensional case. We make a connection between the one-dimensional modified Keller-Segel model (1.3) and certain Boltzmann-like equations used in granular gases and wealth-distribution models; see [17, 20] and the references therein. This connection is due to the fact that (1.3) can be written in Fourier variables such as the referenced Boltzmann equations. The unexpected property is that we show that equation (1.3) is indeed a contraction for the so-called Fourier distance $d_{1}$ defined in (4.3) in Section 4 borrowing the ideas of 17 .

Theorem 1.3. Assume $N=1$ and $\chi<1$. The one-dimensional Keller-Segel system (1.3) is a contraction for the Fourier distance $d_{1}$ in the class of solutions having zero center of mass and finite second moment. The rescaled problem (1.10) is a uniformly strict contraction in the rescaled frame with a contraction factor which does not depend on $\chi$. 


\section{An alternative Proof of the Logarithmic HLS inequality}

2.1. Preliminaries on optimal transport tools. Let $\tilde{\rho}$ and $\rho$ be two density probabilities. According to [13, 25] there exists a convex function $\psi$ whose gradient pushes forward the measure $\tilde{\rho}(a) d a$ onto $\rho(x) d x: \nabla \psi \#(\tilde{\rho}(a) d a)=\rho(x) d x$. This convex function satisfies the Monge-Ampère equation in the weak sense: for any test function $\varphi \in C_{b}\left(\mathbb{R}^{N}\right)$, the following identity holds true:

$$
\int_{\mathbb{R}^{N}} \varphi(\nabla \psi(a)) \tilde{\rho}(a) d a=\int_{\mathbb{R}^{N}} \varphi(x) \rho(x) d x .
$$

Regularity of the transport map is a big issue in general. Here we will use the fact that the Hessian measure $\operatorname{det}_{H} D^{2} \psi(a)$ can be decomposed into an absolutely continuous part $\operatorname{det}{ }_{A} D^{2} \psi(a)$ and a positive singular measure [30, Chapter 4]. Moreover, it is known that a convex function $\psi$ has Aleksandrov second derivative $D_{A}^{2} \psi(a)$ almost everywhere and that $\operatorname{det}_{A} D^{2} \psi(a)=\operatorname{det} D_{A}^{2} \psi(a)$. In particular we have $\operatorname{det}_{H} D^{2} \psi(a) \geq \operatorname{det}{ }_{A} D^{2} \psi(a)$. The formula for the change of variables will be important when dealing with the entropy contribution. For any measurable function $U$ bounded below such that $U(0)=0$, we have [26, 30.

$$
\int_{\mathbb{R}^{N}} U(\tilde{\rho}(x)) d x=\int_{\mathbb{R}^{N}} U\left(\frac{\rho(a)}{\operatorname{det}{ }_{A} D^{2} \psi(a)}\right) \operatorname{det}_{A} D^{2} \psi(a) d a .
$$

In fact this paper will only be concerned with the one-dimensional case and the two-dimensional radial case. The complexity of Brenier's transport problem dramatically reduces in both cases. In dimension one, the transport map $\psi^{\prime}$ is a nondecreasing function; therefore it is differentiable a.e. and it has a countable number of jump singularities. The singular part of the positive measure $\psi^{\prime \prime}$ corresponds to having holes in the support of the density $\rho$. Also, the Aleksandrov second derivative of $\psi$ coincides with the absolutely continuous part of the positive measure $\psi^{\prime \prime}$ that will be denoted by $\psi_{\mathrm{ac}}^{\prime \prime}$. Moreover, the a.e. representative $\psi^{\prime}$ can be chosen to be the distribution function of the measure $\psi^{\prime \prime}$ and it is of bounded variation locally, with lateral derivatives existing at all points, and therefore we can always write for all $a<b$,

$$
\psi^{\prime}(b)-\psi^{\prime}(a)=\int_{(a, b]} d \psi^{\prime \prime}(x) \geq \int_{a}^{b} \psi_{\mathrm{ac}}^{\prime \prime}(x) d x
$$

for a well-chosen representative of $\psi^{\prime}$.

In the two-dimensional radial case, Brenier's map can be expressed in terms of the one-dimensional transport between the densities $2 \pi \tilde{\rho}(a) a^{d-1} d a$ and $2 \pi \rho(r) r^{d-1} d r$ that we call $\psi^{\prime}$. The determinant of the Hessian is given by

$$
\operatorname{det}_{H} D^{2} \psi(a)=\frac{1}{2 a} \frac{d}{d a}\left(\psi^{\prime}\right)^{2}(a),
$$

where the derivative of $\left(\psi^{\prime}\right)^{2}$ has to be understood in the distributional sense. Here, we have made a little abuse of notation by writing the Hessian measure already in radial coordinates. Since $\psi^{\prime}$ is again nondecreasing, similar properties as for the one-dimensional case hold. More precisely, we can choose the a.e. representative $\left(\psi^{\prime}\right)^{2}(a)$ such that it corresponds to the distribution function of the positive measure $2 a \operatorname{det}_{H} D^{2} \psi(a)$ in the interval $[0, \infty)$. Thus, we can write

$$
\left(\psi^{\prime}\right)^{2}(a)=2 \int_{0}^{a} b \operatorname{det}_{H} D^{2} \psi(b) d b .
$$


The following lemma will be used to estimate the interaction contribution in the free energy and in the evolution of the Wasserstein distance. For notational convenience, we denote the convex combination of $a$ and $b$ by $[a, b]_{t}=(1-t) a+t b$.

Lemma 2.1. Let $K:(0, \infty) \rightarrow \mathbb{R}$ be an increasing and strictly concave function. Then, for any $(a, b)$,

$$
K\left(\frac{\psi^{\prime}(b)-\psi^{\prime}(a)}{b-a}\right) \geq \int_{0}^{1} K\left(\psi_{\mathrm{ac}}^{\prime \prime}\left([a, b]_{t}\right)\right) d t .
$$

Equality is achieved in (2.5) if and only if the distributional derivative of the transport map $\psi^{\prime \prime}$ is a constant function.

Analogously in the two-dimensional radially symmetric case we deduce for any $a>0$,

$$
K\left(\frac{\left(\psi^{\prime}\right)^{2}(a)}{a^{2}}\right) \geq \int_{0}^{a} K\left(\operatorname{det}_{A} D^{2} \psi(b)\right) \frac{2 b}{a^{2}} d b .
$$

Equality is achieved in (2.6) if and only if $\psi^{\prime}$ is a multiple of the identity.

Proof. We have on the one hand (2.3). We next use the concavity of $K$ and Jensen's inequality to conclude the proof. Equality occurs if $\psi^{\prime \prime}$ is absolutely continuous and if $\psi_{\mathrm{ac}}^{\prime \prime}$ is constant. In the two-dimensional case we use $\operatorname{det}{ }_{H} D^{2} \psi(a) \geq \operatorname{det}{ }_{A} D^{2} \psi(a)$ and (2.4), and we proceed analogously.

Optimal transport is a powerful tool for reducing functional inequalities onto pointwise inequalities (e.g. matrix inequalities). We highlight for example the seminal paper by McCann [26], where the displacement convexity issue for some energy functional is reduced to the concavity of $\operatorname{det}^{1 / N}$. We also refer to the works of Barthe [2, 3, and Cordero-Erausquin et al. [18]. We require simple pointwise inequalities which are extensions of the classical Jensen's inequality.

Lemma 2.2. Let $\alpha, \beta, \gamma>0$. For any measurable function $u:(0,1) \rightarrow(0,+\infty)$ :

$$
\begin{array}{r}
\alpha\left(\int_{0}^{1} u(t) d t\right)^{-\gamma}-\beta\left(\int_{0}^{1} u(t) d t\right)^{\gamma} \leq(\alpha+\beta) \int_{0}^{1}(u(t))^{-\gamma} d t-2 \beta, \\
\alpha \log \left(\int_{0}^{1} u(t) d t\right)+\beta\left(\int_{0}^{1} u(t) d t\right)^{2} \geq(\alpha+2 \beta)\left(\int_{0}^{1} \log (u(t)) d t\right)+\beta .
\end{array}
$$

In both cases equality holds true if and only if $u(t)=1$ a.e.

Proof. We have by Jensen's inequality,

$$
(\alpha+\beta)\left(\int_{0}^{1} u(t) d t\right)^{-\gamma} \leq(\alpha+\beta) \int_{0}^{1}(u(t))^{-\gamma} d t
$$

thus

$$
\begin{aligned}
\alpha\left(\int_{0}^{1} u(t) d t\right)^{-\gamma}-\beta\left(\int_{0}^{1} u(t) d t\right)^{\gamma} \leq(\alpha+\beta) \int_{0}^{1}(u(t))^{-\gamma} d t-2 \beta \\
+\beta\left[2-\left(\int_{0}^{1} u(t) d t\right)^{\gamma}-\left(\int_{0}^{1} u(t) d t\right)^{-\gamma}\right]
\end{aligned}
$$

We conclude the proof since the quantity between brackets is nonpositive $\left(\forall X X^{-1}\right.$ $+X \geq 2$ ). Equality arises if and only if $u$ is almost everywhere constant and 
$\int_{0}^{1} u(t) d t=1$. On the other hand, the Jensen inequality applied to the log function directly gives

$$
\begin{gathered}
\alpha \log \left(\int_{0}^{1} u(t) d t\right)+\beta\left(\int_{0}^{1} u(t) d t\right)^{2} \geq(\alpha+2 \beta)\left(\int_{0}^{1} \log (u(t)) d t\right)+\beta \\
+\beta\left[-1-2 \log \left(\int_{0}^{1} u(t) d t\right)+\left(\int_{0}^{1} u(t) d t\right)^{2}\right] .
\end{gathered}
$$

We conclude the proof since the quantity between brackets is nonnegative $\left(\forall X X^{2} \geq\right.$ $1+2 \log X)$. Again, equality arises if and only if $u$ is almost everywhere constant and $\int_{0}^{1} u(t) d t=1$.

2.2. The one-dimensional case. The novelty in this section is contained in the proof of the Logarithmic HLS inequality. We show below that the Logarithmic HLS inequality can be recovered as a consequence of Jensen's inequality.

Our first lemma is a reformulation of the Euler-Lagrange equation for the extremal function (1.7).

Lemma 2.3 (Characterization of extremal functions). The critical profile $\mu$ (1.9) satisfies the following identity:

$$
\mu(p)=\int_{\mathbb{R}} \int_{0}^{1} \mu(p-t q) \mu(p-t q+q) d t d q .
$$

In the subcritical regime $\chi<1$, the stationary solution to (1.10), denoted by $\nu$, satisfies the following identity:

$$
\nu(p)=\int_{\mathbb{R}} \int_{0}^{1}\left(\chi+\frac{|q|^{2}}{2}\right) \nu(p-t q) \nu(p-t q+q) d t d q .
$$

Proof. The critical profile $\mu$ is, in particular, a solution to (1.7). Actually, the formulation (2.9) is equivalent to integrating once the equation satisfied by the critical profile. We integrate equation (1.7) against some test function $\varphi$ :

$$
\begin{aligned}
\int_{\mathbb{R}} \varphi^{\prime}(p) \mu(p) d p & =2 \iint_{\mathbb{R} \times \mathbb{R}} \frac{\varphi(x)}{x-y} \mu(x) \mu(y) d x d y \\
& =\iint_{\mathbb{R} \times \mathbb{R}} \frac{\varphi(x)-\varphi(y)}{x-y} \mu(x) \mu(y) d x d y \\
& =\iint_{\mathbb{R} \times \mathbb{R}} \int_{0}^{1} \varphi^{\prime}\left([x, y]_{t}\right) \mu(x) \mu(y) d t d x d y \\
& =\int_{\mathbb{R}} \varphi^{\prime}(p)\left\{\int_{\mathbb{R}} \int_{0}^{1} \mu(p-t q) \mu(p-t q+q) d t d q\right\} d p
\end{aligned}
$$

where we have finally used the change of variables: $(x, y) \mapsto\left(p=[x, y]_{t}, q=y-x\right)$. This holds true for any derivative $\varphi^{\prime}$, so we obtain identity (2.9) up to a constant. Since both sides of (2.9) have mass one, the constant is zero. The identity (2.10) is obtained in a similar way by integrating over the one-dimensional stationary equation version of (1.10).

Proof of Theorem 1.1. Let us take the critical profile $\mu$ and any other density $\rho$ for which the log-interaction kernel integral is well-defined. We consider the optimal map $\psi^{\prime}$ pushing $\mu(a) d a$ onto $\rho(x) d x$. Applying the change of variables formula (2.2) 
for $x=\psi^{\prime}(a)$ and using the definition of push-forward (2.1), the functional $\mathcal{F}$ can be rewritten as follows:

$$
\begin{aligned}
\mathcal{F}[\rho]-\mathcal{F}[\mu] & =\int_{\mathbb{R}} \log \left(\frac{\mu(a)}{\psi_{\mathrm{ac}}^{\prime \prime}(a)}\right) \mu(a) d a+\iint_{\mathbb{R} \times \mathbb{R}} \log \left|\psi^{\prime}(a)-\psi^{\prime}(b)\right| \mu(a) \mu(b) d a d b-\mathcal{F}[\mu] \\
& =-\int_{\mathbb{R}} \log \left(\psi_{\mathrm{ac}}^{\prime \prime}(a)\right) \mu(a) d a+\iint_{\mathbb{R} \times \mathbb{R}} \log \left(\frac{\psi^{\prime}(a)-\psi^{\prime}(b)}{a-b}\right) \mu(a) \mu(b) d a d b .
\end{aligned}
$$

Using Lemma 2.1 for $K=\log z$, which is increasing and concave, we deduce

$$
\begin{aligned}
\mathcal{F}[\rho]-\mathcal{F}[\mu] & \geq-\int_{\mathbb{R}} \log \left(\psi_{\mathrm{ac}}^{\prime \prime}(p)\right) \mu(p) d p+\iint_{\mathbb{R} \times \mathbb{R}} \int_{0}^{1} \log \left(\psi_{\mathrm{ac}}^{\prime \prime}\left([a, b]_{t}\right)\right) \mu(a) \mu(b) d t d a d b \\
& =\int_{\mathbb{R}} \log \left(\psi_{\mathrm{ac}}^{\prime \prime}(p)\right)\left\{-\mu(p)+\int_{\mathbb{R}} \int_{0}^{1} \mu(p-t q) \mu(p-t q+q) d t d q\right\} d p=0,
\end{aligned}
$$

due to the identity (2.9) for the critical profile. We conclude that $\mathcal{F}[\rho] \geq \mathcal{F}[\mu]$, which is equivalent to the Logarithmic HLS inequality. Equality arises if and only if the transport map $\psi^{\prime \prime}$ is a constant function corresponding to dilations of $\mu$.

It is possible to extend Theorem 1.1 to the rescaled energy $\mathcal{F}_{\text {resc }}$ (1.11).

Theorem 2.4 (Logarithmic HLS inequality with a quadratic confinement). Assume $N=1,2$ and $\chi<1$. The functional $\mathcal{F}_{\text {resc }}$ is bounded from below and possesses a unique minimizer in the set of probability densities with zero center of mass.

Proof of Theorem 2.4. We give details of the proof only in the one-dimensional case. The case $N=2$ is analogous. The key point consists in replacing Jensen's inequality with the convex-like inequality (2.8). We use the fact that the center of mass is zero to double the number of variables: $\int_{\mathbb{R}}|x|^{2} \rho(x) d x=\frac{1}{2} \iint_{\mathbb{R} \times \mathbb{R}}|x-y|^{2} \rho(x) \rho(y) d x d y$. Define $\psi^{\prime}$ to be the map that pushes forward $\nu(a) d a$ onto $\rho(x) d x$. Applying (2.2) for $x=\psi^{\prime}(a)$ and using (2.3) as in Lemma 2.1. the functional $\mathcal{F}_{\text {resc }}$ reads as:

$$
\begin{aligned}
& \mathcal{F}_{\text {resc }}[\rho]-\mathcal{F}_{\text {resc }}[\nu] \\
& =-\int_{\mathbb{R}} \log \left(\psi_{\mathrm{ac}}^{\prime \prime}(a)\right) \nu(a) d a+\iint_{\mathbb{R} \times \mathbb{R}} \log \left(\frac{\psi^{\prime}(a)-\psi^{\prime}(b)}{a-b}\right) \nu(a) \nu(b) d a d b \\
& \quad+\frac{1}{4} \iint_{\mathbb{R} \times \mathbb{R}}\left|\psi^{\prime}(a)-\psi^{\prime}(b)\right|^{2} \nu(a) \nu(b) d a d b-\frac{1}{2} \int_{\mathbb{R}}|a|^{2} \nu(a) d a \\
& \geq-\int_{\mathbb{R}} \log \left(\psi_{\mathrm{ac}}^{\prime \prime}(a)\right) \nu(a) d a-\frac{1}{2} \int_{\mathbb{R}}|a|^{2} \nu(a) d a \\
& +\iint_{\mathbb{R} \times \mathbb{R}}\left(\chi \log \left(\int_{0}^{1} \psi_{\mathrm{ac}}^{\prime \prime}\left([a, b]_{t}\right) d t\right)+\frac{|a-b|^{2}}{4}\left(\int_{0}^{1} \psi_{\mathrm{ac}}^{\prime \prime}\left([a, b]_{t}\right) d t\right)^{2}\right) \nu(a) \nu(b) d a d b .
\end{aligned}
$$

Applying inequality (2.8) with $\alpha=\chi$ and $\beta=|a-b|^{2} / 4$ we deduce

$$
\begin{aligned}
& \mathcal{F}_{\text {resc }}[\rho]-\mathcal{F}_{\text {resc }}[\nu] \\
& \geq-\int_{\mathbb{R}} \log \left(\psi_{\mathrm{ac}}^{\prime \prime}(p)\right) \nu(p) d p-\frac{1}{2} \int_{\mathbb{R}}|a|^{2} \nu(a) d a+\frac{1}{4} \iint_{\mathbb{R} \times \mathbb{R}}|a-b|^{2} \nu(a) \nu(b) d a d b \\
& \quad+\iint_{\mathbb{R} \times \mathbb{R}} \int_{0}^{1}\left(\chi+\frac{|a-b|^{2}}{2}\right) \log \left(\psi_{\mathrm{ac}}^{\prime \prime}([a, b] t)\right) \nu(a) \nu(b) d t d a d b \\
& =\int_{\mathbb{R}} \log \left(\psi_{\mathrm{ac}}^{\prime \prime}(p)\right)\left\{-\nu(p)+\int_{q \in \mathbb{R}} \int_{0}^{1}\left(\chi+\frac{|q|^{2}}{2}\right) \nu(p-t q) \nu(p-t q+q) d t d q\right\} d p .
\end{aligned}
$$


We conclude from characterization (2.10) that $\mathcal{F}_{\text {resc }}[\rho]-\mathcal{F}_{\text {resc }}[\nu] \geq 0$. Equality arises if and only if $\psi^{\prime \prime}(a)=1$ a.e. This guarantees uniqueness of the minimizer.

2.3. The two-dimensional case. We restrict ourselves to radially symmetric functions in the two-dimensional case due to a decreasing rearrangement [24, 4, 16]. We recall Newton's theorem for the Poisson potential: the field induced by a radially symmetric distribution of masses outside a given ball is equivalent to the field induced by a point at the center of the ball 24. Equivalently it reads

$$
\frac{1}{2} \int_{0}^{2 \pi} \log \left(r^{2}+s^{2}-2 r s \cos (\theta)\right) d \theta=2 \pi \log \max (r, s) .
$$

As a consequence we can rewrite the functional $\mathcal{F}$ in a simple way under radial symmetry:

$$
\frac{1}{2 \pi} \mathcal{F}[\rho]=\frac{1}{2} \int_{\mathbb{R}_{+}} \rho(r) \log (\rho(r)) r d r+\chi \int_{\mathbb{R}_{+}} \rho(r) M[\rho](r) \log (r) r d r .
$$

The following characterizations are direct consequences of (1.8) and the radial stationary equation version of (1.10).

Lemma 2.5 (Characterization of extremal functions under radial symmetry). The critical profile $\mu$ for $N=2$ satisfies the following identity:

$$
\frac{1}{2} \mu(b)=2 \int_{b}^{+\infty} \mu(a) M[\mu](a) \frac{1}{a} d a .
$$

In the subcritical regime $\chi<1$, the radially-symmetric stationary solution to (1.10) satisfies the following identity:

$$
\frac{1}{2} \nu(b)=\int_{b}^{+\infty} \nu(a)\left(2 \chi M[\nu](a) \frac{1}{a}+a\right) d a .
$$

We are now ready to examine the Logarithmic HLS inequality in the twodimensional radial setting.

Proof of Theorem 1.1. We proceed as in the one-dimensional case. Let us take the critical profile $\mu$ and any other density $\rho$ for which the log-interaction kernel integral is well-defined. We consider the radial optimal map $\psi^{\prime}$ pushing $\mu(a) a d a$ to $\rho(r) r d r$. We apply the change of variables formulas (2.2) and (2.1) to get

$\frac{1}{2 \pi} \mathcal{F}[\rho]=\frac{1}{2} \int_{\mathbb{R}_{+}} \mu(a) \log \left(\frac{\mu(a)}{\operatorname{det}{ }_{A} D^{2} \psi(a)}\right) a d a+2 \int_{\mathbb{R}_{+}} \mu(a) M[\mu](a) \log \left(\psi^{\prime}(a)\right) a d a$,

where we have used $M[\rho](r)=M[\mu](a)$. We have consequently,

$$
\begin{aligned}
\frac{1}{2 \pi} \mathcal{F}[\rho]-\frac{1}{2 \pi} \mathcal{F}[\mu]= & -\frac{1}{2} \int_{\mathbb{R}_{+}} \mu(a) \log \left(\operatorname{det}_{A} D^{2} \psi(a)\right) a d a \\
& +\int_{\mathbb{R}_{+}} \mu(a) M[\mu](a) \log \left(\frac{\left(\psi^{\prime}\right)^{2}(a)}{a^{2}}\right) a d a .
\end{aligned}
$$


The last contribution of (2.14) can be evaluated using Lemma 2.1.

$$
\begin{aligned}
& \int_{\mathbb{R}_{+}} \mu(a) M[\mu](a) \log \left(\int_{0}^{a}\left(\operatorname{det}_{H} D^{2} \psi(b)\right) \frac{2 b}{a^{2}} d b\right) a d a \\
& \geq \int_{\mathbb{R}_{+}} \int_{0}^{a} \mu(a) M[\mu](a) \log \left(\operatorname{det}_{A} D^{2} \psi(b)\right) \frac{2 b}{a} d b d a \\
& =\int_{\mathbb{R}_{+}} \log \left(\operatorname{det}_{A} D^{2} \psi(b)\right)\left\{2 \int_{b}^{+\infty} \mu(a) M[\mu](a) \frac{1}{a} d a\right\} b d b .
\end{aligned}
$$

We obtain from the characterization (2.12), $\mathcal{F}[\rho] \geq \mathcal{F}[\mu]$. Again equality occurs if and only if the transport map $\psi^{\prime}$ is a multiple of the identity.

We skip the proof of Theorem 2.4 in the case $N=2$ since it is analogous to the case $N=1$.

\section{Exponential CONVERGENCE TOWARdS THE SELF-SIMILAR PROFILE}

3.1. The one-dimensional case. To illustrate the strategy of the proof of Theorem 1.2. we show a formal computation in the critical case $\chi=1$. Up to our knowledge, the regularity of solutions under very weak assumptions is still an open problem. In particular it is not known whether the solutions satisfy the identity (1.6) or not. So the following computation is questionable because the velocity field $\partial_{x}(\log \rho(t, x)+2 \log |x| * \rho(t, x))$ is not clearly defined in $L^{2}(\rho(t, x) d x)$.

We compute formally the evolution of the Wasserstein distance to one of the equilibria (1.9) in the critical case $\chi=1$. Notice that equilibria are infinitely far from each other with respect to the Wasserstein distance 8 . We denote by $\partial_{x} \phi(t, x)$ the map that pushes forward $\rho(t, x) d x$ onto $\mu(a) d a$, and by $\partial_{a} \psi(t, a)$ the reverse map. Using the gradient flow structure with respect to $W_{2}$, one obtains the following formula for the derivative of $F(t)=W_{2}(\rho(t), \mu)^{2}$; see [30, Chapter 8] and 1]:

$$
\begin{aligned}
\frac{F^{\prime}(t)}{2} & =\int_{\mathbb{R}}\left(\partial_{x} \phi(t, x)-x\right)\left(\partial_{x}(\log \rho(t, x)+2 \log |x| * \rho(t, x))\right) \rho(t, x) d x \\
& =-\int_{\mathbb{R}} \partial_{x x}^{2} \phi(t, x) \rho(t, x) d x+\iint_{\mathbb{R} \times \mathbb{R}} \frac{\partial_{x} \phi(t, x)-\partial_{x} \phi(t, y)}{x-y} \rho(t, x) \rho(t, y) d x d y \\
& =-\int_{\mathbb{R}}\left(\partial_{a a}^{2} \psi(t, a)\right)^{-1} \mu(a) d a+\iint_{\mathbb{R} \times \mathbb{R}}\left(\frac{\partial_{a} \psi(t, a)-\partial_{a} \psi(t, b)}{a-b}\right)^{-1} \mu(a) \mu(b) d a d b \\
& \leq-\int_{\mathbb{R}}\left(\partial_{a a}^{2} \psi(t, a)\right)^{-1} \mu(a) d a+\iint_{\mathbb{R} \times \mathbb{R}} \int_{0}^{1}\left(\partial_{a a}^{2} \psi\left(t,[a, b]_{s}\right)\right)^{-1} \mu(a) \mu(b) d s d a d b .
\end{aligned}
$$

We recognize the characterization (2.9). Hence, we have at least formally $F^{\prime}(t) \leq 0$, where we have used Jensen's inequality with the convex function $X^{-1}$.

The same strategy is valid in the subcritical case $\chi<1$ for which we know that solutions are regular enough to ensure the validity of the computations. As a matter of fact, the density $\rho(t, x)$ is everywhere positive and thus $\partial_{a a}^{2} \psi$ is absolutely continuous. On the other hand the dissipation of energy is well-defined and the dissipation estimate (1.6) holds true [12. 
Proof of Theorem 1.2. We compute the evolution of $F(t)=W_{2}(\rho(t), \nu)^{2}$. We denote by $\partial_{x} \phi(t, x)$ the map that pushes forward $\rho(t, x) d x$ onto $\nu(a) d a$. We use that the center of mass is zero to double the number of variables as in Theorem 2.4, and thus we get

$$
\begin{aligned}
\frac{F^{\prime}(t)}{2}= & \int_{\mathbb{R}}\left(\partial_{x} \phi(t, x)-x\right)\left(\partial_{x}\left(\log \rho(t, x)+2 \chi \log |x| * \rho(t, x)+\frac{|x|^{2}}{2}\right)\right) \rho(t, x) d x \\
= & -\int_{\mathbb{R}} \partial_{x x}^{2} \phi(t, x) \rho(t, x) d x+\chi \iint_{\mathbb{R} \times \mathbb{R}} \frac{\partial_{x} \phi(t, x)-\partial_{x} \phi(t, y)}{x-y} \rho(t, x) \rho(t, y) d x d y \\
& -\frac{1}{2} \iint_{\mathbb{R} \times \mathbb{R}}\left(\partial_{x} \phi(t, x)-\partial_{x} \phi(t, y)\right)(x-y) \rho(t, x) \rho(t, y) d x d y \\
& +2 \int_{\mathbb{R}} \partial_{x} \phi(t, x) x \rho(t, x) d x+1-\chi-\int_{\mathbb{R}}|x|^{2} \rho(t, x) d x .
\end{aligned}
$$

We rewrite each contribution using the reverse transport map $\partial_{a} \psi$. We recall that the second moment of the stationary state is explicitly given by (1.13):

$$
\begin{aligned}
\frac{F^{\prime}(t)}{2}= & -\int_{\mathbb{R}}\left(\partial_{a a}^{2} \psi(t, a)\right)^{-1} \nu(a) d a \\
& +\iint_{\mathbb{R} \times \mathbb{R}}\left[\chi\left(\frac{\partial_{a} \psi(t, a)-\partial_{a} \psi(t, b)}{a-b}\right)^{-1}\right. \\
& \left.\quad-\frac{|a-b|^{2}}{2}\left(\frac{\partial_{a} \psi(t, a)-\partial_{a} \psi(t, b)}{a-b}\right)\right] \nu(a) \nu(b) d a d b \\
& +2 \int_{\mathbb{R}} a \partial_{a} \psi(t, a) \nu(a) d a+\int_{\mathbb{R}}|a|^{2} \nu(a) d a-\int_{\mathbb{R}}\left|\partial_{a} \psi(t, a)\right|^{2} \nu(a) d a \\
\leq & -\int_{\mathbb{R}}\left|\partial_{a} \psi(t, a)-a\right|^{2} \nu(a) d a+2 \int_{\mathbb{R}}|a|^{2} \nu(a) d a-\int_{\mathbb{R}}\left(\partial_{a a}^{2} \psi(t, a)\right)^{-1} \nu(a) d a \\
& +\int_{\mathbb{R}^{2}}\left[\left(\chi+\frac{|a-b|^{2}}{2}\right) \int_{0}^{1}\left(\partial_{a a}^{2} \psi\left(t,[a, b]_{s}\right)\right)^{-1} d s-|a-b|^{2}\right] \nu(a) \nu(b) d a d b,
\end{aligned}
$$

where we have applied (2.7) for $\gamma=1, \alpha=\chi$ and $\beta=|a-b|^{2} / 2$. Using that the center of mass is zero to simplify the previous expression and defining $A(t)$ by

$$
\int_{\mathbb{R}}\left(\partial_{a a}^{2} \psi(t, p)\right)^{-1}\left\{-\nu(p)+\int_{\mathbb{R}} \int_{0}^{1}\left(\chi+\frac{|q|^{2}}{2}\right) \nu(p-t q) \nu(p-t q+q) d t d q\right\} d p
$$

we deduce that $F^{\prime}(t)+2 F(t) \leq 2 A(t)=0$ by the characterization (2.10).

3.2. The two-dimensional radially-symmetric case. Proving convergence towards a self-similar profile in the rescaled logarithmic case under radial symmetry goes as previously.

Proof of Theorem 1.2. Define $\partial_{r} \phi^{\prime}(t, r)$ to be the map that pushes forward $\rho(r) r d r$ onto $\nu(a) a d a$, and $\partial_{a} \psi^{\prime}(t, a)$ to be the reverse map. We compute again the evolution 
of the Wasserstein distance $F(t)=W_{2}(\rho(t), \nu)^{2}$ :

$$
\begin{aligned}
\frac{F^{\prime}(t)}{2}= & \int_{\mathbb{R}_{+}}\left(\partial_{r} \phi(t, r)-r\right)\left(\frac{1}{2} \partial_{r} \log \rho(t, r)+2 \chi M[\rho](t, r) \frac{1}{r}+r\right) \rho(t, r) r d r \\
= & \frac{1}{2} \int_{\mathbb{R}_{+}} r \partial_{r} \phi(t, r) \partial_{r} \rho(t, r) d r+2 \chi \int_{\mathbb{R}_{+}} \partial_{r} \phi(t, r) M[\rho](t, r) \rho(t, r) d r \\
& +\int_{\mathbb{R}_{+}} \partial_{r} \phi(t, r) \rho(t, r) r^{2} d r+1-\chi-\int_{\mathbb{R}_{+}} \rho(t, r) r^{3} d r \\
=-\frac{1}{2} \int_{\mathbb{R}_{+}}\left(\frac{\partial_{r} \phi(t, r)}{r}+\partial_{r r}^{2} \phi(t, r)\right) \rho(t, r) r d r & \int_{\mathbb{R}_{+}}\left(\partial_{r} \phi(t, r)^{2}\right)^{1 / 2} M[\rho](t, r) \rho(t, r) d r-\int_{\mathbb{R}_{+}} \partial_{r} \phi(t, r) \rho(t, r) r^{2} d r \\
+ & \quad 2 \chi \int_{\mathbb{R}_{+}} \partial_{r} \phi(t, r) \rho(t, r) r^{2} d r+\int_{\mathbb{R}_{+}} \nu(a) a^{3} d a-\int_{\mathbb{R}_{+}} \rho(t, r) r^{3} d r \\
\leq- & F(t)+2 \int_{\mathbb{R}_{+}} \nu(a) a^{3} d a-\int_{\mathbb{R}_{+}}\left(\frac{1}{\operatorname{det} D^{2} \psi(b)}\right)^{-1 / 2} \nu(b) b d b \\
+ & \int_{\mathbb{R}_{+}}\left[2 \chi M[\nu](a)\left(\int_{0}^{a} \operatorname{det} D^{2} \psi(b) \frac{2 b}{a^{2}} d b\right)^{-1 / 2}\right] \nu(a) a d a .
\end{aligned}
$$

The last step in the inequality is a consequence of the arithmetic and geometric means inequality: $\left(\partial_{r} \phi(t, r) / r+\partial_{r r}^{2} \phi(t, r)\right) \geq 2\left(\partial_{r} \phi(t, r) \partial_{r r}^{2} \phi(t, r) / r\right)^{1 / 2}$. Next we use Lemma 2.2 to handle the interaction contribution. More precisely, we choose $\gamma=1 / 2, \alpha=2 \chi M[\nu](a)$ and $\beta=a^{2}$. One finally gets $F^{\prime}(t)+2 F(t) \leq 0$, where the characterization (2.13) was used. This concludes the proof.

\section{Contraction in the one-Dimensional CaSe}

The aim of this section is to point out the peculiar structure of the modified onedimensional Keller-Segel system (1.3). First we derive an equivalent form for (1.3) which makes a surprising connection with the one-dimensional Boltzmann homogeneous equation. We denote the Fourier transform as $\hat{\rho}(t, \xi)=\int_{\mathbb{R}} e^{-i \xi x} \rho(t, x) d x$.

Lemma 4.1. Equation (1.3) can be rewritten in Fourier variables as

$$
\partial_{t} \hat{\rho}(t, \xi)=|\xi|^{2}\left(-\hat{\rho}(t, \xi)+\chi \int_{0}^{1} \hat{\rho}(t, \sigma \xi) \hat{\rho}(t,(1-\sigma) \xi) d \sigma\right) .
$$

According to (4.1) the information propagates from lower to higher frequencies. The evolution of $\hat{\rho}(t, \xi)$ requires the knowledge of lower frequencies $\left|\xi^{\prime}\right|<|\xi|$ due to the integral contribution. This is of particular importance for designing a numerical scheme. Indeed there is no loss of information after truncation of the frequency box.

Remark 4.2 (Analogy with 1D Boltzmann). It is worth mentioning that the integral operator on the right-hand side of (4.1) is reminiscent of the homogeneous Boltzmann equations in 1D used for granular gases [17] or wealth distribution models [20] in Fourier variables. 


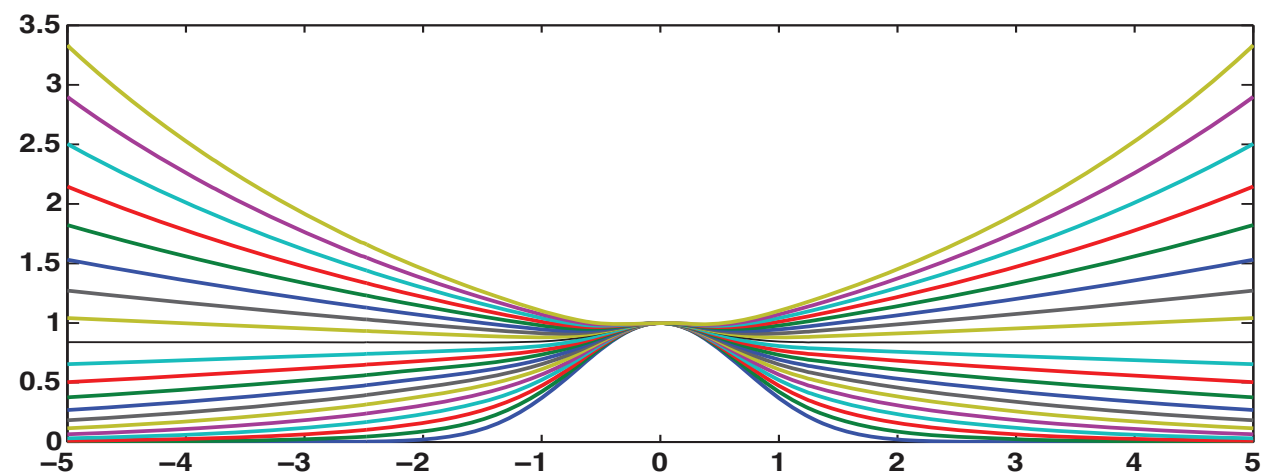

Figure 1. Simulation of (4.1) in the supercritical case $\chi>1$ for successive times. The solution at the blow-up time $T$ is plotted in bold dark lines. Clearly the solution is no longer the Fourier transform of a probability measure for $t>T$.

Remark 4.3 (Evidence for blowup in the supercritical case). We can directly notice the occurrence of blowup when $\chi>1$ from (4.1). Observe that for $|\xi| \ll 1$, the right-hand side is equivalent to

$$
\partial_{t} \hat{\rho}(t, \xi) \sim|\xi|^{2}\left(-\hat{\rho}(t, 0)+\chi \hat{\rho}(t, 0)^{2}\right)=|\xi|^{2}(-1+\chi) .
$$

This argues in favor of blowup at low modes although misleadingly. We have plotted in Figure 1 a numerical simulation of (4.1) in the supercritical case. Observe that blowup arises for $|\xi| \gg 1$, contrary to the misleading heuristics (4.2). The integrodifferential equation (4.1) makes perfect sense even in the supercritical regime $\chi>1$ after the first blow-up event. However the outcoming function $\hat{\rho}(t, \xi)$ is no longer the Fourier transform of a probability measure. In fact the blow-up time coincides with the formation of the first Dirac mass, namely when the frequency distribution $\widehat{\rho}(t, \xi)$ is flat at infinity. This contradictory intuition is similar to the proof of the blowup based on the virial identity: the second momentum provides information at infinity but is used to prove blowup, which is a local behaviour.

Proof of Lemma 4.1. We test equation (1.3) against $\exp (-i \xi x)$ :

$$
\begin{aligned}
\frac{\partial}{\partial t} \widehat{\rho}(t, \xi) & =\int_{\mathbb{R}}\left(\partial_{x x} \rho(t, x)+2 \chi \partial_{x}\left(\rho(t, x)\left(\partial_{x} \log |x|\right) * \rho(t, x)\right)\right) e^{-i \xi x} d x \\
& =-|\xi|^{2} \hat{\rho}(t, \xi)+\chi i \xi \iint_{\mathbb{R} \times \mathbb{R}} \rho(t, x) \frac{e^{-i \xi x}-e^{-i \xi y}}{x-y} \rho(t, y) d x d y \\
& =-|\xi|^{2} \hat{\rho}(t, \xi)+\chi|\xi|^{2} \iint_{\mathbb{R} \times \mathbb{R}} \rho(t, x)\left(\int_{0}^{1} e^{-i \xi[x, y]_{\sigma}} d \sigma\right) \rho(t, y) d x d y \\
= & -|\xi|^{2} \hat{\rho}(t, \xi)+\chi|\xi|^{2} \int_{0}^{1}\left(\int_{\mathbb{R}} \rho(t, x) e^{-i(1-\sigma) \xi x} d x\right)\left(\int_{\mathbb{R}} \rho(t, y) e^{-i \sigma \xi y} d y\right) d \sigma,
\end{aligned}
$$

which gives the desired formulation.

Recall the definition of Fourier distances [17] as they have been introduced for the analysis of the Boltzmann equation. 
Definition 4.4 (Fourier distances). Let $\rho_{1}, \rho_{2}$ be two probability measures having the same center of mass. The $d_{1}$-distance is defined as follows:

$$
d_{1}\left(\rho_{1}, \rho_{2}\right)=\sup _{\xi \neq 0}\left\{|\xi|^{-1}\left|\hat{\rho}_{1}(\xi)-\hat{\rho}_{2}(\xi)\right|\right\} .
$$

Proof of Theorem 1.3. First notice that the supremum in (4.3) is attained in $\mathbb{R} \backslash\{0\}$. Clearly we have $\left|\hat{\rho}_{1}(\xi)-\hat{\rho}_{2}(\xi)\right| \leq 2$ and

$$
\hat{\rho}_{1}(\xi)-\hat{\rho}_{2}(\xi) \sim\left(\int_{\mathbb{R}}|x|^{2}\left[\rho_{1}(x)-\rho_{2}(x)\right] d x\right)|\xi|^{2} / 2 \text { as } \xi \rightarrow 0 .
$$

Let $\rho_{1}, \rho_{2}$ be two solutions to (1.3). We denote $F(t)=d_{1}\left(\rho_{1}(t), \rho_{2}(t)\right)$ and $h(t, \xi)=$ $|\xi|^{-1}\left(\hat{\rho}_{1}(t, \xi)-\hat{\rho}_{2}(t, \xi)\right)$. Multiplying the difference between equations (4.1) by $\operatorname{sign}(h(t, \xi))$, we get $\partial_{t}|h(t, \xi)|=|\xi|^{2}\left(-|h(t, \xi)|+\chi \operatorname{sign}\left(\hat{\rho}_{1}(t, \xi)-\hat{\rho}_{2}(t, \xi)\right) A(t, \xi)\right)$, where

$$
A(t, \xi)=|\xi|^{-1} \int_{0}^{1} \hat{\rho}_{1}(t, \sigma \xi) \hat{\rho}_{1}(t,(1-\sigma) \xi) d \sigma-|\xi|^{-1} \int_{0}^{1} \hat{\rho}_{2}(t, \sigma \xi) \hat{\rho}_{2}(t,(1-\sigma) \xi) d \sigma .
$$

The self-attraction terms are treated by [17, Th. 6.3], and since $\left\|\hat{\rho}_{i}(t)\right\|_{\infty} \leq 1$,

$$
|A(t, \xi)| \leq d_{1}\left(\rho_{1}(t), \rho_{2}(t)\right) \int_{0}^{1}(\sigma+(1-\sigma)) d \sigma=F(t) .
$$

We obtain finally $\partial_{t}|h(t, \xi)| \leq|\xi|^{2}(-|h(t, \xi)|+\chi F(t))$, from which we deduce

$$
\limsup _{\epsilon \rightarrow 0^{+}} \frac{F(t+\epsilon)-F(t)}{\epsilon} \leq(\chi-1)\left(\liminf _{\epsilon \rightarrow 0^{+}}\left|\xi^{*}(t+\epsilon)\right|^{2}\right) F(t),
$$

where $\left|\xi^{*}(t)\right|$ denotes the lowest frequency moduli for which the supremum is attained in $F(t)=\sup |h(t, \xi)|$. Therefore we get a contraction estimate as soon as $\chi<1$. There is no explicit rate since we do not know how to control $\left|\xi^{*}(t)\right|$ from below. We also obtain a uniform strict contractivity in self-similar variables. The Keller-Segel equation (1.10) in Fourier variables can be written as follows:

$$
\partial_{t} \hat{\rho}(t, \xi)=|\xi|^{2}\left(-\hat{\rho}(t, \xi)+\chi \int_{0}^{1} \hat{\rho}(t, \sigma \xi) \hat{\rho}(t,(1-\sigma) \xi) d \sigma\right)-\xi \partial_{\xi} \hat{\rho}(t, \xi) .
$$

Proceeding as above and integrating along the characteristics, we deduce

$$
\limsup _{\epsilon \rightarrow 0^{+}} \frac{F(t+\epsilon)-F(t)}{\epsilon} \leq-F(t)+(\chi-1)\left(\liminf _{\epsilon \rightarrow 0^{+}}\left|\xi^{*}(t+\epsilon)\right|^{2}\right) F(t) .
$$

Hence the one-dimensional Keller-Segel equation (4.1) is a contraction with rate 1 with respect to the Fourier distance $d_{1}$.

\section{ACKNOWLEDGMENTS}

Both authors are supported by the bilateral France-Spain project FR2009-0019. The second author is partially supported by the projects MTM2008-06349-C0303 DGI-MCI (Spain) and 2009-SGR-345 from AGAUR-Generalitat de Catalunya. Both authors thank CRM-Barcelona, where part of this work was done during the stay of the first author as part of the thematic program in mathematical biology in 2009 . 


\section{REFERENCES}

[1] L. A. Ambrosio, N. Gigli, G. Savaré, Gradient flows in metric spaces and in the space of probability measures, Lectures in Mathematics, Birkhäuser, 2005. MR2129498 (2006k:49001)

[2] F. Barthe, Inégalités de Brascamp-Lieb et convexité, C. R. Math. Acad. Sci. Paris 324 (1997), no. 8, 885-888. MR1450443(98a:26022)

[3] F. Barthe, On a reverse form of the Brascamp-Lieb inequality, Invent. Math. 134 (1998), no. 2, 335-361. MR1650312 (99i:26021)

[4] W. Beckner, Sharp Sobolev inequalities on the sphere and the Moser-Trudinger inequality, Ann. of Math. (2) 138 (1993), no. 1, 213-242. MR1230930 (94m:58232)

[5] P. Biler, G. Karch, P. Laurençot, T. Nadzieja, The $8 \pi$-problem for radially symmetric solutions of a chemotaxis model in the plane, Math. Methods Appl. Sci. 29 (2006), no. 13, 1563-1583. MR2249579 (2007f:35156)

[6] P. Biler, T. Nadzieja, Existence and nonexistence of solutions for a model of gravitational interaction of particles I, Colloq. Math. 66 (1994), no. 2, 319-334. MR.1268074 (95b:35223)

[7] A. Blanchet, V. Calvez, J. A. Carrillo, Convergence of the mass-transport steepest descent scheme for the subcritical Patlak-Keller-Segel model, SIAM J. Numer. Anal. 46 (2008), no. 2, 691-721. MR2383208 (2009a:35113)

[8] A. Blanchet, E. Carlen, J. A. Carrillo, Functional inequalities, thick tails and asymptotics for the critical mass Patlak-Keller-Segel model, J. Funct. Anal., to appear.

[9] A. Blanchet, J. A. Carrillo, P. Laurençot, Critical mass for a Patlak-Keller-Segel model with degenerate diffusion in higher dimensions, Calc. Var. Partial Differential Equations 35 (2009), no. 2, 133-168. MR2481820(2010e:35149)

[10] A. Blanchet, J. A. Carrillo, N. Masmoudi, Infinite time aggregation for the critical PatlakKeller-Segel model in $\mathbb{R}^{2}$, Comm. Pure Appl. Math. 61 (2008), no. 10, 1449-1481. MR2436186 (2009d:35132)

[11] A. Blanchet, J. Dolbeault, M. Escobedo, J. Fernández, Asymptotic behaviour for small mass in the two-dimensional parabolic-elliptic Keller-Segel model, J. Math. Anal. Appl. 361 (2010), no. 2, 533-542. MR2568716 (2010m:35190)

[12] A. Blanchet, J. Dolbeault, B. Perthame, Two-dimensional Keller-Segel model: Optimal critical mass and qualitative properties of the solutions, Electron. J. Differential Equations (2006), no. 44, 32 pp. (electronic). MR2226917(2007e:35277)

[13] Y. Brenier, Polar factorization and monotone rearrangement of vector-valued functions, Comm. Pure Appl. Math. 44 (1991), no. 4, 375-417. MR1100809 (92d:46088)

[14] V. Calvez, B. Perthame, M. Sharifi Tabar, Modified Keller-Segel system and critical mass for the log interaction kernel, Stochastic analysis and partial differential equations, 45-62, Contemp. Math., 429, Amer. Math. Soc., Providence, RI, 2007. MR2391528 (2009d:60272)

[15] E. Carlen, J.A. Carrillo, M. Loss, Hardy-Littlewood-Sobolev inequalities via fast diffusion flows, Proc. Natl. Acad. Sci. USA 107 (2010), no. 46, 19696-19701. MR2745814

[16] E. Carlen, M. Loss, Competing symmetries, the logarithmic HLS inequality and Onofri's inequality on $S^{n}$, Geom. Funct. Anal. 2 (1992), no. 1, 90-104. MR1143664 (93b:58170)

[17] J. A. Carrillo, G. Toscani, Contractive probability metrics and asymptotic behavior of dissipative kinetic equations, Riv. Mat. Univ. Parma 6 (2007), 75-198. MR2355628(2008i:82094)

[18] D. Cordero-Erausquin, B. Nazaret, C. Villani, A mass-transportation approach to sharp Sobolev and Gagliardo-Nirenberg inequalities, Adv. Math. 182 (2004), no. 2, 307-332. MR2032031 (2005b:26023)

[19] J. Dolbeault, B. Perthame, Optimal critical mass in the two-dimensional Keller-Segel model in $\mathbb{R}^{2}$, C. R. Math. Acad. Sci. Paris 339 (2004), no. 9, 611-616. MR2103197 (2005h:35189)

[20] B. Düring, D. Matthes, G. Toscani, A Boltzmann-type approach to the formation of wealth distribution curves, Riv. Mat. Univ. Parma 1 (2009), 199-261. MR2597795

[21] R. Jordan, D. Kinderlehrer, F. Otto, The variational formulation of the Fokker-Planck equation, SIAM J. Math. Anal. 29 (1998), no. 1, 1-17. MR1617171 (2000b:35258)

[22] N. I. Kavallaris, P. Souplet, Grow-up rate and refined asymptotics for a two-dimensional Patlak-Keller-Segel model in a disk, SIAM J. Math. Anal. 40 (2008/09), no. 5, 1852-1881. MR2471903 (2010a:35121)

[23] E. F. Keller, L. A. Segel, Initiation of slime mold aggregation viewed as an instability, J. Theor. Biol. 26 (1970), no. 3, 399-415. 
[24] E. H. Lieb, M. Loss, Analysis, Vol. 14, Amer. Math. Soc, Providence, 1997. MR1415616 (98b:00004)

[25] R. J. McCann, Existence and uniqueness of monotone measure-preserving maps, Duke Math. J. 80 (1995), no. 2, 309-323. MR.1369395 (97d:49045)

[26] R. J. McCann, A convexity principle for interacting gases, Adv. Math. 128 (1997), no. 1, 153-179. MR1451422 (98e:82003)

[27] T. Nagai, Blow-up of radially symmetric solutions to a chemotaxis system, Adv. Math. Sci. Appl. 5 (1995), no. 2, 581-601 MR:1361006 (96j:35249)

[28] F. Otto, The geometry of dissipative evolution equations: the porous medium equation, Comm. Partial Differential Equations 26 (2001), no. 1-2, 101-174. MR.1842429 (2002j:35180)

[29] C. S. Patlak, Random walk with persistence and external bias, Bull. Math. Biophys. 15 (1953), 311-338. MR0081586 (18:424f)

[30] C. Villani, Topics in optimal transportation, Graduate Studies in Mathematics, Vol. 58, Amer. Math. Soc, Providence, RI, 2003. MR1964483 (2004e:90003)

École Normale Supérieure de Lyon, UMR CNRS 5669 "Unité de Mathématiques Pures et Appliquées", 46 allée d'Italie, F-69364 Lyon Cedex 07, France

E-mail address: vincent.calvez@ens-lyon.fr

Institució Catalana de Recerca i Estudis Avançats and Departament de Matemàtiques, Universitat Autònoma de Barcelona, E-08193 Bellaterra, Spain

E-mail address: carrillo@mat.uab.es 